\title{
Thin lenses of asymmetric power
}

\author{
W F Harris* and R D van Gool**
}

Department of Optometry, University of Johannesburg, PO Box 524, Auckland Park, 2006 South

Africa

*<wharris@uj.ac.za>

$* *<$ rdvg@mweb.co.za $>$

Received 3 July 2008; revised version accepted 9 March 2009

\begin{abstract}
It is generally supposed that thin systems, including refracting surfaces and thin lenses, have powers that are necessarily symmetric. In other words they have powers which can be represented as symmetric dioptric power matrices and in the familar spherocylindrical form used in optometry and ophthalmology. This paper shows that this is not correct and that it is indeed possible for a thin system to have a power that is not symmetric and which cannot be expressed in spherocylindrical form. Thin systems of asymmetric power are illustrated
\end{abstract}

by means of a thin lens that is modelled with small prisms and is chosen to have a dioptric power matrix that is antisymmetric. Similar models can be devised for a thin system whose dioptric power matrix is any $2 \times 2$ matrix. Thus any power, symmetric, asymmetric or antisymmetric, is possible for a thin system. In this sense our understanding of the power of thin systems is now complete.

Key words: dioptric power matrix, symmetric power, asymmetric power, antisymmetric power, prism

\section{Introduction}

It is generally believed that it is not possible for a refracting surface, a thin lens or any other thin system to have a dioptric power that is asymmetric. The purpose of this paper is to challenge that belief and describe thin systems which do, indeed, have asymmetric powers.

We begin by defining symmetric powers in terms of the symmetric dioptric power matrix and traditional ways of representing such powers in optometry. We then examine asymmetric powers (they have no counterpart in traditional optometric or ophthalmological thought) for lenses that are thick. Fick's and Long's generalization of Prentice's equation for prismatic effect is then used to model a thin lens of a particular asymmetric power. This shows that asymmetric powers are possible for thin systems in general. We show the effect of a thin lens of a particular asymmetric power on rays traversing it. Finally we touch on possible areas for future research

\section{Symmetric powers}

Traditionally powers in optometry and ophthalmology are expressed in three common ways, spherocylindrical, for example, $3-2 \times 40$ and $12 \times 130$, principal meridional, for example $3\{40\} 1\{130\}$, and crossed cylindrical, for example $1 \times 403 \times 130$. The principal meridians (represented by the 40 and 130) 
are at right angles. All four of these quantitative representations mean exactly the same thing; they mean the power expressed as the dioptric power matrix

$$
\mathbf{F}=\left(\begin{array}{ll}
2.174 & 0.985 \\
0.985 & 1.826
\end{array}\right) \text { D. }
$$

This matrix representation of power, and the method of obtaining it from the spherocylindrical power, was first presented by Fick ${ }^{1}$, as was pointed out by Diepes and Blendowske ${ }^{2,3}$. It was later and independently pointed out by Long ${ }^{4}$. In general the dioptric power matrix has the form

$$
\mathbf{F}=\left(\begin{array}{ll}
f_{11} & f_{12} \\
f_{21} & f_{22}
\end{array}\right) \mathrm{D}
$$

in which the off-diagonal entries, $f_{12}$ and $f_{21}$, are necessarily equal and, so, $\mathbf{F}$ is necessarily a symmetric matrix. Every power expressed in any of the traditional forms described above can be represented as a symmetric dioptric power matrix. (The reverse is also true.) Hence, because they all have representations as symmetric dioptric power matrices, all traditional powers can be described as symmetric powers.

\section{Asymmetric powers}

Formally a matrix $\mathbf{F}$ is symmetric if $\mathbf{F}^{\mathrm{T}}=\mathbf{F}$ where $\mathbf{F}^{\mathrm{T}}$ is the matrix transpose of $\mathbf{F}$. This is equivalent for $2 \times 2$ matrices to the statement that the off-diagonal entries of $\mathbf{F}$ are equal. A matrix that is not symmetric is called asymmetric; its off-diagonal entries differ. If $\mathbf{F}^{\mathrm{T}}=-\mathbf{F}$ then $\mathbf{F}$ is called antisymmetric; its off-diagonal entries are equal in magnitude but opposite in sign and its diagonal entries are zeros. With the exception of null power (plano) all antisymmetric powers are also asymmetric. (Null power is both symmetric and antisymmetric but not asymmetric. Antisymmetric matrices are also called skew-symmetric. For more on these matrices the reader is referred elsewhere. ${ }^{5}$ )

The very structure of the dioptric power matrix, with its four entries (Equations 1 and 2), hints at the possibility that there may be a whole new class of non-traditional powers that are unrecognized in optometry and ophthalmology, a class of powers for which $f_{12} \neq f_{21}$. If they existed they would have asymmetric dioptric power matrices. In other words they would have asymmetric powers. To represent them one would necessarily require, in general, four distinct numbers; three distinct numbers that are sufficient for traditional powers (for example, sphere, cylinder and axis in the spherocylindrical forms) would not be sufficient for representing such powers.

The first asymmetric powers were described by Fick ${ }^{6}$ and later by Keating ${ }^{7,8}$, but these were only for thick lenses. Thin systems had powers that were necessarily symmetric. A thick bitoric lens of reduced thickness $\tau$ and first- and second-surface powers $\mathbf{F}_{1}$ and $\mathbf{F}_{2}$ has power ${ }^{6-8}$

$$
\mathbf{F}=\mathbf{F}_{1}+\mathbf{F}_{2}-\tau \mathbf{F}_{2} \mathbf{F}_{1}
$$

(In keeping with common practice Keating called such powers equivalent powers. The term is redundant, however, and such powers are preferably referred to simply as powers ${ }^{9}$.) While $\mathbf{F}_{1}$ and $\mathbf{F}_{2}$ are traditional powers, that is, they are symmetric, the product $\mathbf{F}_{2} \mathbf{F}_{1}$ in Equation 3 is not symmetric in general. Thus asymmetric powers are possible for thick lenses. But what about thin lenses? For a thin lens one sets $\tau=0$ and Equation 3 becomes

$$
\mathbf{F}=\mathbf{F}_{1}+\mathbf{F}_{2}
$$

Since the sum of symmetric matrices is symmetric it would seem that the power $\mathbf{F}$ of a thin lens is necessarily symmetric.

\section{Prism models of thin lenses}

Ordinary stigmatic (spherical) thin lenses are often thought of in terms of prisms. Figure 1 shows an example. The biconvex lens is represented in crosssection as eight prisms whose apical angles increase in magnitude from zero in the centre to a maximum at the periphery. The base-to-apex direction is radial and outward from the centre. The combination of prisms better approximates the lens the greater the number of component prisms and the smaller their size. Rays through the lens are deflected radially inward. (This assumes that the index of refraction of the lens is greater than the index of the surrounding medium. For the purposes of this paper we shall take the surrounding medium as air. The conclusions hold 
with appropriate modification for any mediums.) The deflection (or prismatic effect) can be calculated by means of Prentice's well-known equation. (A case can be made for the equation to be renamed Imbert's equation. ${ }^{10-12}$ )

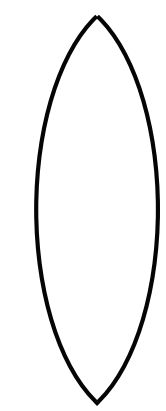

(a)

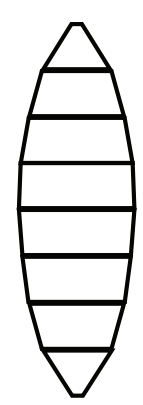

(b)
Figure 1. Cross-section of a lens (a) modeled in terms of prisms (b).

Fick's ${ }^{13}$ and Long's ${ }^{4}$ generalization (Equation 5) of Prentice's equation is not restricted to spherical lenses but holds for all symmetric (spherical, cylindrical and spherocylindrical) powers. Consider a point $\mathrm{P}$ on a thin lens of (symmetric) power $\mathbf{F}$ and optical centre $\mathrm{O}$ (Figure 2). Let $\mathrm{P}$ have position vector $\mathbf{y}$ relative to $\mathrm{O}$. A ray traversing the lens at $\mathrm{P}$ has deflection $\mathbf{p}$ given by

$$
\mathbf{p}=-\mathbf{F y}
$$

the generalized form of Prentice's equation. The same equation is given by Campbell. ${ }^{14,15}$ In the neighbourhood of point $P$ the lens behaves as a small prism with apex-to-base direction the same as the direction of $\mathbf{p}$ and whose deflection is given by $p$, the magnitude of p. The prism's apical angle $b$ is given simply by

$$
b=p /\left(n-n_{0}\right)
$$

where $n$ and $n_{0}$ are the indices of refraction of the prism and the surrounding medium respectively.

We can imagine approximating a lens of any given dioptric power $\mathbf{F}$ by putting together small prisms whose geometry and orientation at each point is defined by Equations 5 and 6. Alternatively we can imagine building up any thin lens by laying out an array of small prisms of the appropriate apical angle and orientation. That we could do for any given symmetric power $\mathbf{F}$. But there is no reason why we should not be able to do the same for any given asymmetric matrix $\mathbf{F}$ as well.

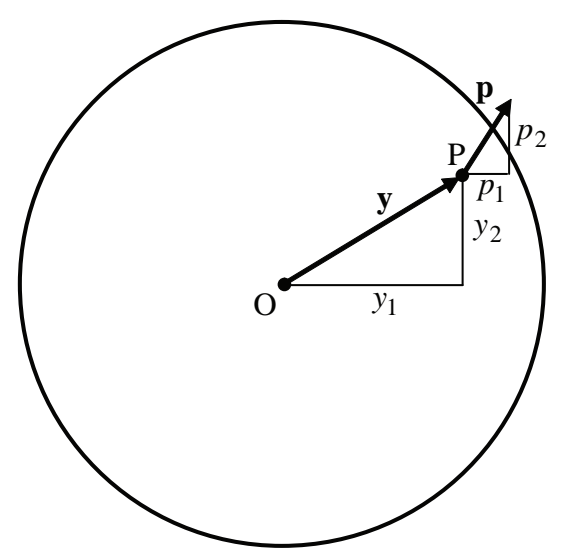

Figure 2. A thin lens is viewed along its axis. Point $\mathrm{P}$ on a thin lens has position vector $\mathbf{y}$ with respect to the optical centre $\mathrm{O}$ of the lens. It has horizontal and vertical components $y_{1}$ and $y_{2}$ respectively. The deflection or prismatic effect at $\mathrm{P}$ is represented by vector $\mathbf{p}$ with components $p_{1}$ and $p_{2}$. In particular a ray parallel to the longitudinal axis would emerge with inclination p. In Figure $4 \mathbf{p}$ is perpendicular to $\mathbf{y}$ and points in a clockwise sense about $\mathrm{O}$.

\section{A thin lens of antisymmetric power}

\section{Consider the power}

$$
\mathbf{F}=-\mathbf{L} \mathrm{D}
$$

where

$$
\mathbf{L}:=\left(\begin{array}{cc}
0 & 1 \\
-1 & 0
\end{array}\right) .
$$

(We note that $\mathbf{L}$ is the negative of the matrix represented by $\mathbf{L}$ in recent papers ${ }^{16-18}$. This makes $\mathbf{L}$ the same matrix as used in the definition of $2 \times 2$ symplectic and Hamiltonian matrices and brings it in line with usage elsewhere ${ }^{9,14,15,19}$.) Because matrix $\mathbf{L}$ is antisymmetric $\mathbf{F}$ is an antisymmetric power. Let us suppose a thin lens of that power could be made. We think of building the lens up of many small square prisms (Figure 3). (There is no requirement that the prisms be square; squares are chosen merely for convenience of illustration. For any practical realization of the lens it is quite possible that other geometries would be preferable.) From Equation 5 we see that a prism with centre at a point with position vector $y$ must have deflection 


$$
\mathbf{p}=-\left(\begin{array}{cc}
0 & -1 \\
1 & 0
\end{array}\right)\left(\begin{array}{l}
y_{1} \\
y_{2}
\end{array}\right)
$$

that is,

$$
\mathbf{p}=\left(\begin{array}{c}
y_{2} \\
-y_{1}
\end{array}\right),
$$

where the components of $\mathbf{y}$ are in metres. Equation 10 shows that the deflection has a direction that is clockwise from y. Thus the apex-to-base directions of all the prisms must be arranged circumferentially and clockwise as represented symbolically in Figure 3. The magnitudes of the apical angles grow linearly outwards from the centre as is directly evident from Equation 5; this is also represented symbolically in Figure 3 by means of bars (for the bases) of increasing thickness.

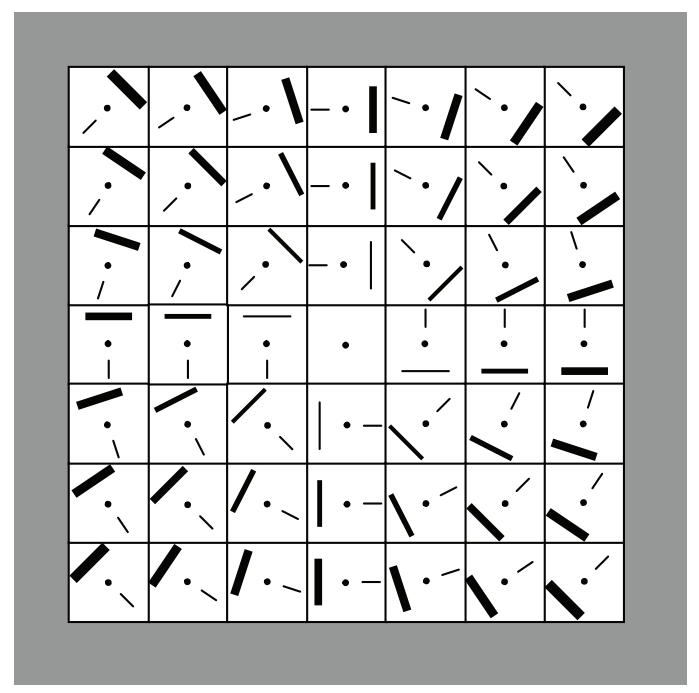

Figure 3 An array of small square prisms making up a square lens of divergence $\mathbf{L ~ D}$ and dioptric power $-\mathbf{L} \mathbf{D}$. There are three marks on each prism: a dot marks the geometrical centre, a short line segment marks the direction of the apex and a longer line segment represents the base. The central square represents a prism of zero apical angle. The apical angle increases linearly with distance from the centre as represented symbolically by line segments of increasing thickness. The apex-to-base direction is circumferential and clockwise. Squares and their particular arrangement are chosen for illustrative purposes; other shapes and arrays are perfectly possible and are likely to be preferable in any actual realization of the lens.

\section{Rays through a thin lens of antisymmetric power}

We suppose now that parallel rays are incident perpendicularly onto this side of the putative lens at the dots in Figure 3. They emerge from the lens on the other side with the twisted appearance shown in Figure 4. Following the rays downstream we see that they splay outward as they twist. The same happens if we follow the projections of these rays in the upstream direction (not shown in the figure). No point or line focus is formed anywhere, either as a real fo-. cus downstream or a virtual focus upstream. Figure 4 is the same as illustrated by Campbell ${ }^{14}$ for a thick system (see his Figure 6) and described as one that 'cannot be realized by a surface or a lens'. Campbell's statement holds for smooth surfaces; the lens of Figure 3 has a rough surface. The arrows represent a vector field ${ }^{14}$.

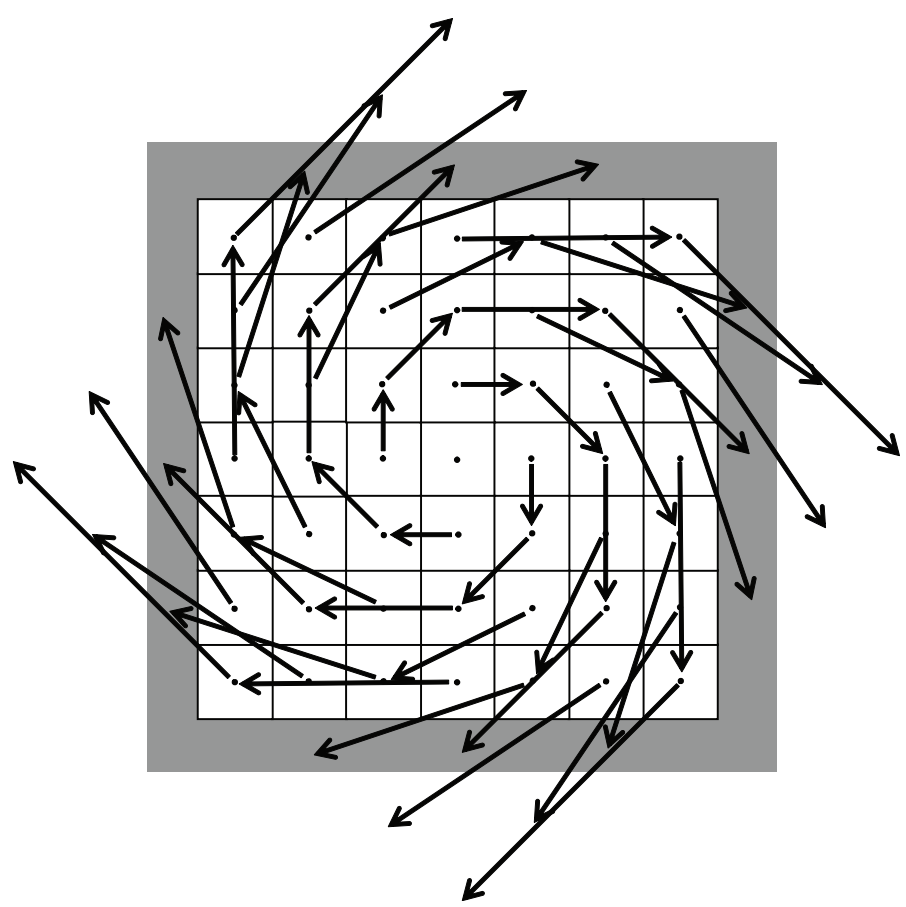

Figure 4 Rays traversing the lens of Figure 3. The dots in the centres of the squares represent incident ray segments orthogonal to and on this side of the lens. The arrows represent the emergent segments on the other side of the lens. The emergent segments exhibit a clockwise swirling pattern as one travels with the light. The central dot represents the optical axis. The chirality is reversed in the case of a thin lens of power $\mathbf{L} D$. The arrows can also be viewed as constituting a vector field.

A deflected but untwisted beam of light with a constant square cross-section emerges from each small prism. The central beam is undeflected. Figure 5 shows successive cross-sections downstream from the lens. The differing deflections cause the individual component beams gradually to part company, as one follows them downstream, leaving light-free gaps in between. The result is a twisted composite 
beam made up of untwisted and gradually separating component beams as shown from (a) to (d) in Figure 5. In (c) the composite beam has twisted clockwise through $45^{\circ}$ and the dark spaces between the component beams have grown to form a checkerboard pattern. The blue square moves horizontally to the right; the orange square moves vertically downward; and the green square moves right and down. A photograph of a simple model is shown in Figure 6 although the deflections have been exaggerated.

Let us suppose that the square lens in Figure 3 has a side of length $70 \mathrm{~mm}$, is made of plastic with index 1.5 and is in air. The individual prisms then have sides of length $10 \mathrm{~mm}$. Consider first the first prism to the right of the central one; it is the prism that transmits the beam shown orange in Figure 5. According to Equation 10 it deflects the light by

$$
\mathbf{p}=\left(\begin{array}{c}
0 \\
-0.01
\end{array}\right)
$$

that is, by 0.01 downward. That is equivalent to 1 prism dioptre (pd) or about $0.57^{\circ}$. The apical angle of the prism is about $1.1^{\circ}$ (Equation 6). The base is down. The same figures apply to the other three prisms adjacent to the central one except that the base directions are as shown in Figure 3. Doubling these numbers gives the properties of the second prism to the right of the central one; multiplying them by 3 gives the properties of the third prism to the right. Thus the apical angle increases outwards from the centre along the middle row, and along the middle column, approximately as follows: $1.1^{\circ}, 2.3^{\circ}$ and $3.4^{\circ}$. It follows from these numbers that Figure 5(b) represents the cross-section of the compound beam in a transverse plane that is $0.5 \mathrm{~m}$ beyond the lens; (c) and (d) are cross-sections at 1.0 and $1.5 \mathrm{~m}$ respectively. (Instead of a length of $1.5 \mathrm{~m}$ the box in Figure 6 has a length of about $30 \mathrm{~cm}$. Thus the angles in Figure 6 are exaggerated by the factor about 5 . Alternatively one can regard the figure as a representation of the rays downstream from a thin lens of power approximately $-5 \mathbf{L ~ D}$ when the rays incident onto the thin lens are all perpendicular to it.) For every square of light in Figure 5(b) there is a dark square that is a quarter of its area. Thus the area of the cross-section of the compound beam has expanded by a factor of about $\frac{5}{4}$ Similarly one sees that the area of the cross-section in (c) has enlarged by a factor of about $\frac{8}{4}$ (double) and in (d) by a factor of about $\frac{13}{4}$. The square roots of these numbers give the length of the side of the square cross-section of the beam at each transverse plane.

\section{Lenses of any power, symmetric or asymmetric}

It is perfectly possible to construct an array of prisms of the sort we have been examining. And it is apparent that the array would model a thin lens of power -L D; it does to light, at least approximately, what a thin lens of the power concerned would do to light. In the same way one could model a thin lens of power $\mathbf{F}$ where $\mathbf{F}$ is any $2 \times 2$ matrix. The model would improve as the number of prisms increased and their size decreased although effects in physical optics would impose the practical limitation that the size of the prisms should not be very much less than a millimeter. The lens would have a rough surface and could not be polished smooth. The optical quality of the lens could not be as good as in the case of a conventional lens but one expects that it might approach that of a Fresnel lens.

The power $(-\mathbf{L} \mathbf{D})$ used here to illustrate the argument was chosen because, being antisymmetric, it represents a particular asymmetric power that could not be further from being symmetric. It corresponds in particular to a divergence of $\mathbf{L} \mathbf{D}$ and results in a right-handed twist to the bundle of rays traversing the lens. (Divergence is the negative of dioptric power. ${ }^{9,20}$ ) $\mathbf{L} \mathrm{D}$ is the building block, as it were, that makes asymmetric powers asymmetric. In a sense it is the unit of what is responsible for asymmetry. It differs from the divergence of conventional lenses by as much as is possible. This is made clear if we expand the power $\mathbf{F}$ as ${ }^{9,14,18}$

$\mathbf{F}=F_{\mathrm{I}} \mathbf{I}+F_{\mathrm{J}} \mathbf{J}+F_{\mathrm{K}} \mathbf{K}+F_{\mathrm{L}} \mathbf{L}$

where

$\mathbf{I}:=\left(\begin{array}{ll}1 & 0 \\ 0 & 1\end{array}\right)$,

is an identity matrix,

$$
\begin{aligned}
& \mathbf{J}:=\left(\begin{array}{cc}
1 & 0 \\
0 & -1
\end{array}\right), \\
& \mathbf{K}:=\left(\begin{array}{ll}
0 & 1 \\
1 & 0
\end{array}\right)
\end{aligned}
$$


and $\mathbf{L}$ is defined by Equation 8. $\mathbf{I}, \mathbf{J}, \mathbf{K}$ and $\mathbf{L}$ units represent an orthonormal basis for dioptric power. Equation 11 can be written ${ }^{19}$

$$
\mathbf{F}=\mathbf{F}_{\text {symm }}+\mathbf{F}_{\text {antisymm }}
$$

where

$$
\mathbf{F}_{\mathrm{symm}}:=F_{\mathrm{I}} \mathbf{I}+F_{\mathrm{J}} \mathbf{J}+F_{\mathrm{K}} \mathbf{K}
$$

and

$$
\mathbf{F}_{\text {antisymm }}:=F_{\mathrm{L}} \mathbf{L} \text {. }
$$

Traditional powers, being all symmetric, have a null antisymmetric component. Antisymmetric powers have a null symmetric component.

The power $-\mathbf{L} \mathbf{D}$ used in the illustration here implies $F_{\mathrm{L}}=-1 \mathrm{D}$. Clearly any antisymmetric power could be obtained simply by scaling up the apical angles of the prisms accordingly and possibly reversing the apex-to-base direction. It follows from Equation 15 that one could model a thin lens of any power with the symmetric component on one surface and the antisymmetric component on the other; one surface could be conventional (toric or spherical, say) and the other Fresnel-like.

\section{Concluding remarks}

The traditional view of dioptric power is that it is a concept with three degrees of freedom. This is exemplified by the three numbers (sphere, cylinder and axis) required to specify a power in the usual spherocylindrical representation. The mathematical structure of the concept (the $2 \times 2$ dioptric power matrix), however, hints that the concept should really have four degrees of freedom. This paper has shown how that can be the case for a thin system (the four coefficients in Equation 11 for example).

It is indeed possible to have a thin system whose dioptric power is not of the conventional kind, and that the power can be asymmetric. It is the fourth term in Equation 11 that is responsible for the asymmetry. Such powers have dioptric power matrices in which the off-diagonal entries differ and they cannot be expressed in spherocylindrical form. (They can, if a fourth term is added. ${ }^{19}$ ) In fact a thin system may (a)
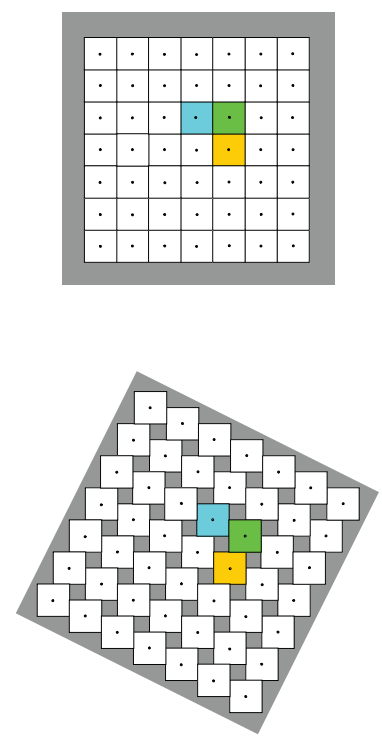

(c)

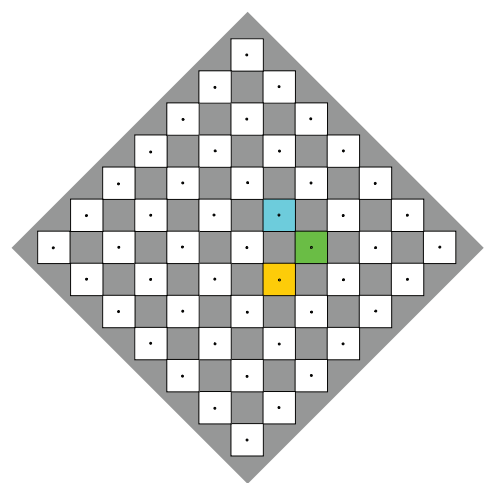

(d)

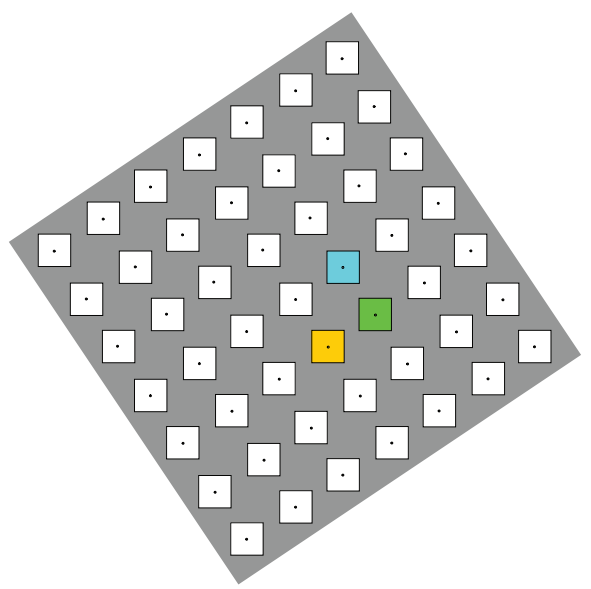

Figure 5 Each small prism with its square aperture (a), except the central one, defines a deflected beam of light with the same square cross-section. The beam defined by the central prism is undeflected. Deflection moves the cross-sections away from each other opening up gaps in which there is no light as shown in successive transverse planes (b) $0.5 \mathrm{~m}$, (c) $1.0 \mathrm{~m}$ and (d) $1.5 \mathrm{~m}$ downstream from the lens. The pattern as a whole retains a square cross-section that turns clockwise and enlarges as one follows the light downstream. In (c) it has rotated $45^{\circ}$ clockwise relative to the lens. The individual squares of light that make up the pattern are themselves not twisted. The blue square moves to the right; the orange square moves downward; and green square moves right and down. 
have any $2 \times 2$ matrix as dioptric power matrix. The particular example used in this paper is that of a thin lens of antisymmetric power, a particular asymmetric power with null symmetric component. It differs from a conventional symmetric power as much as any power can. It is the purest form of powers of this non-traditional class. In general a thin system of asymmetric power has a symmetric (traditional) and an antisymmetric part (Equation 15). In a sense its power would lie between the two extremes of symmetric power and antisymmetric power.

Although we have thought here largely in terms of lenses one can readily apply the same thinking to a single refracting surface as well.

There is nothing special here about square prisms or their arrangement (Figure 3) or the dark patches that arise behind the asymmetric lens described here (in Figure 5(b) to (d) for example). Any lens modelled with small prisms will result in dark patches or overlapping patches of light downstream; the pattern depends on the nature of the prisms and their arrangement. The fact that we have used a square array of prisms is merely because they seem best able to illustrate the issues. Other geometries and other arrays are perfectly possible. In fact considerations of symmetry suggest that squares would not be the best for a practical realization of the lens. Fundamentally all meridians are equivalent. They all have the same torsion $^{21-23}$ and no curvature, in contrast to spherical surfaces in which all meridians have the same curvature but no torsion. Like spherical surfaces there is invariance under rotation about the optical axis. In contrast to conventional refracting surfaces there are no (real) principal meridians.

Nor is there anything special about twisted rays. They are a feature of traditional spherocylindrical lenses. ${ }^{21-23}$

A model of a lens has been constructed here by using Frick's generalization (Equation 5) of Prentice's (or Imbert's) equation. Thus Prentice's equation necessarily applies to lenses of asymmetric power as well. In other words we have generalized Fick's generalization ${ }^{13}$ of Prentice's equation still further: it holds for all thin systems, including those with asymmetric dioptric powers.
The thinking behind this paper has been in terms of the fundamental property divergence ${ }^{9,20} \mathbf{C}$ rather than in terms of the derived property dioptric power F. (One is merely the negative of the other.) However, because of its greater familiarity, we have chosen to present the material in terms of dioptric power instead. This accounts for our use of the power $-\mathbf{L} \mathrm{D}$. The divergence is $\mathbf{L} \mathbf{D}$ and the lens gives the bundle of rays traversing it a right-handed twist. For a thin lens of power $\mathbf{L} \mathbf{D}$ the twist beyond the lens would be left handed.

'Linear optics is an approximation to geometrical optics which is valid when the various angles that enter into consideration are small. ${ }^{24}$ Geometrical optics 'is valid whenever the dimensions of the various apertures are very large when compared to the wavelength of the light and when we do not examine too closely what is happening in the neighborhood of shadows or foci.' 24 This paper uses small prisms to make up the lens, each prism having an aperture much larger than the wavelength of the light. The paper does 'not examine too closely what is happening in the neighborhood of shadows or foci.' Furthermore the angles involved are small. Hence our model satisfies the requirements demanded by linear optics..

However, by their very nature thin lenses of asymmetric (or symmetric) power constructed in the way described above are unlikely to approach conventional lenses in optical quality. They might approach the quality of Fresnel lenses. On the other hand asymmetric powers may turn out to be realizable in other ways, possibly with the use of future materials.

Power, for systems that are thick or thin, can now be recognized fundamentally as a concept with four degrees of freedom. The last term in Equation 11 is null in the case of conventional powers and only three degrees of freedom remain, the first three terms on the right-hand side of Equation 11. More than that, though, all of the degrees of freedom are accounted for, and, in a sense, our understanding of the concept of dioptric power is now complete.

Although we have shown here that lenses of asymmetric power are a conceptual possibility it remains to be seen whether they are of any practical significance. There is much obvious scope for further research. 


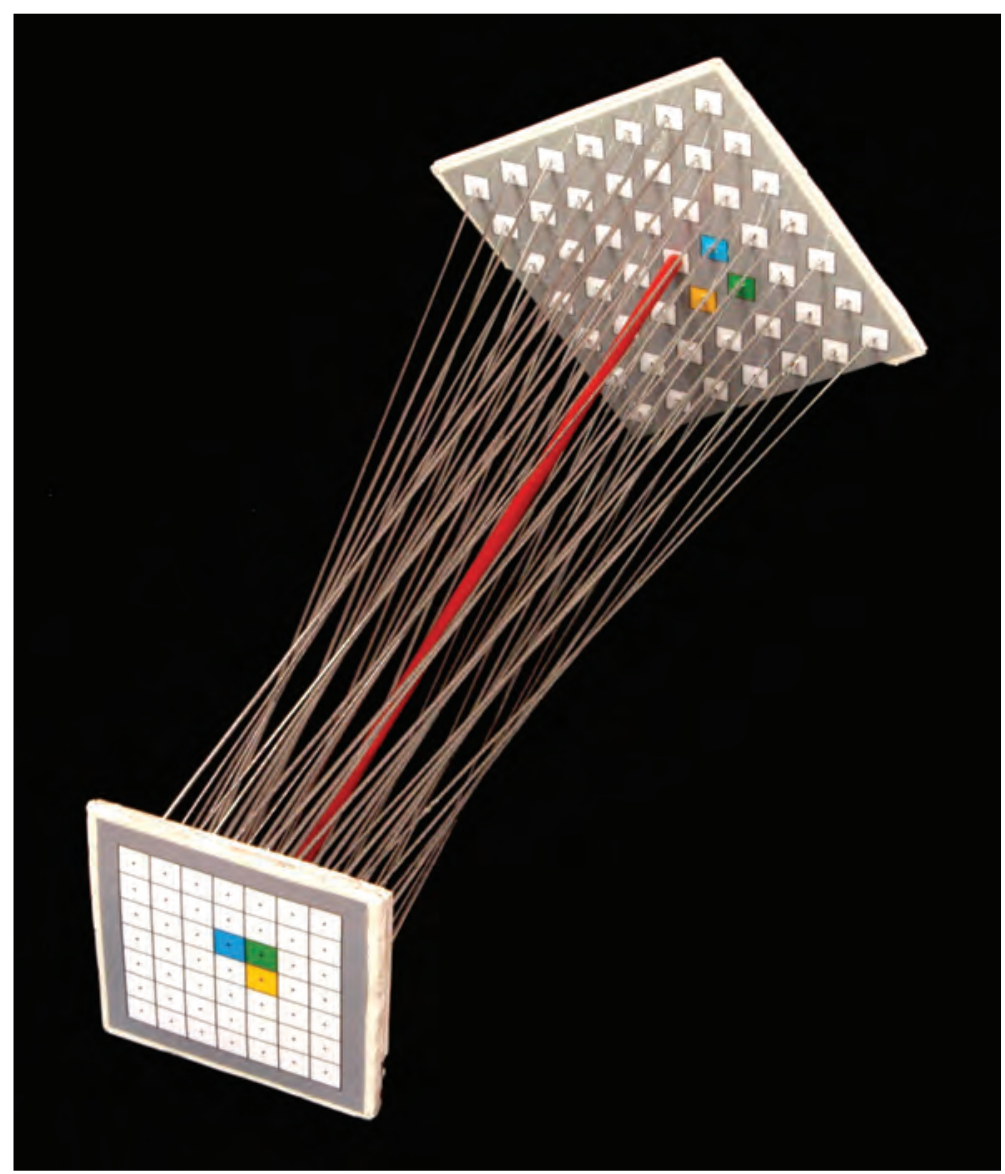

Figure 6 A simple model which shows the rays emerging from the thin lens represented in Figures 3 to 5 . The orange rod at the centre represents the optical axis. The pencil of rays twists in a right-handed sense and expands as one travels away from the lens which is located at the lower left. The incident segments of the rays (not shown) are perpendicular to the lens. The deflections have been exaggerated by a factor of about 5 for clarity. The actual deflections would result from a lens of power $-5 \mathbf{L} \mathrm{D}$ approximately. The model was made by Ms S. Fouché and photographed by C Petker.

\section{Acknowledgements}

The possibility or otherwise of lenses of the type discussed here has been a topic, at least in the background, of discussions that we have been involved in for almost 20 years. All of the following have contributed, in some way, to what is reported in this paper: DJ Malan, A Rubin, S Cronje, CA Blackie, L Joubert, WF Long, MP Keating, LN Thibos, H Abelman, CE Campbell, WDH Gillan, GE MacKenzie, AS Carlson, SD Mathebula, R Blendowske, JR Cardoso. No doubt there are others who ought to be mentioned as well. S Fouché kindly made the model shown in Figure 6 and C Petker kindly did the photography.

\section{References}

1. Fick HH. Fortschrittliche Rechnungsarten in der Augenoptik. Folge 9. Der Augenoptiker 1972 (10) 45-49.

2. Diepes H, Blendowske R. Optik und Technik der Brille. Optische Fachveröffentlichung GmbH, Heidelberg, 2002 477 et seq.

3. Blendowske R. Hans-Heinrich Fick: early contributions to the theory of astigmatic systems. S Afr Optom $2003 \mathbf{8 2}$ 105-110.

4. Long WF. A matrix formalism for decentration problems. Am J Optom Physiol Opt 197653 27-33.

5. Lütkepohl H. Handbook of Matrices. Wiley, Chichester, 1996156 et seq.

6. Fick HH. Fortschrittliche Rechnungsarten in der Augenoptik. Folge 22. Der Augenoptiker 1974 (6) 49-51.

7. Keating MP. A system matrix for astigmatic optical systems: I. Introduction and dioptric power relations. Am J Optom Physiol Opt 198158 810-819.

8. Keating MP. A system matrix for astigmatic optical systems: II. Corrected systems including an astigmatic eye. Am J Optom Physiol Opt 198158 919-929. 
9. Harris WF. Dioptric power: its nature and its representation in three- and four-dimensional space. Optom Vis Sci 1997 74 349-366.

10. Bennett AG. Prismatic effects of spherical and astigmatic lenses: Imbert's pioneer analysis of 1886. Ophthal Physiol Opt 199010 397-398.

11. Imbert A. Calcul de l'effet prismatique des verres décentrés. Annal Oculist 188695 146-153.

12. Harris WF. Effect of spectacle and contact lenses on the effective corneal refractive zone. Clin Exper Optom 2009 92 99-103.

13. Fick HH. Fortschrittliche Rechnungsarten in der Augenoptik. Folge 11. Der Augenoptiker 1972 (12) 60-63.

14. Campbell CE. Ray vector fields. J Opt Soc Am A 199411 618-622.

15. Campbell CE. The refractive group. Optom Vis Sci 1997 74 381-387.

16. Harris WF. Power vectors versus power matrices, and the mathematical nature of dioptric power. Optom Vis Sci 2007 84 1060-1063.

17. Harris WF. Subjective refraction: the mechanism underlying the routine. Ophthal Physiol Opt 200727 594-602.

18. Harris WF. Quantitative analysis of transformed ray transferences of optical systems in a space of augmented Hamiltonian matrices. S Afr Optom 200766 62-67.

19. Harris WF. Keating's asymmetric dioptric power matrices expressed in terms of sphere, cylinder, axis, and asymmetry. Optom Vis Sci 199370 666-667.

20. Harris WF. A unified paraxial approach to astigmatic optics. Optom Vis Sci 199976 480-499.

21. Keating MP. Dioptric power in an off-axis meridian: the torsional component. Am J Optom Physiol Opt 198663 830-838.

22. Harris WF. Signs of surface torsion and torsional dioptric power. Optom Vis Sci 199875 670-673.

23. Harris WF, van Gool RD. Curvital and torsional dioptric power and their polar profiles across meridians. S Afr Optom 199857 3-17.

24. Guillemin V, Sternberg S. Symplectic Techniques in Physics. Cambridge Univ Press, Cambridge, 19845. 